APJ LETTERS

Preprint typeset using $\mathrm{LAT}_{\mathrm{E}} \mathrm{X}$ style emulateapj v. 5/2/11

\title{
FIVE NEW MILLISECOND PULSARS FROM A RADIO SURVEY OF 14 UNIDENTIFIED FERMI-LAT GAMMA-RAY SOURCES
}

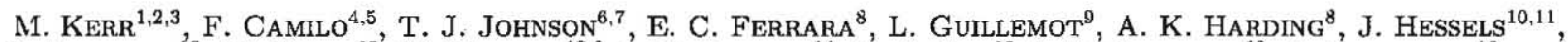 \\ S. Johnston ${ }^{12}$, M. KeIth ${ }^{12}$, M. KRAMER ${ }^{13,9}$ S. M. RANSOM ${ }^{14}$, P. S. RAY ${ }^{15}$ J. E. REYNOLDS ${ }^{12}$, J. SARKISSIAN ${ }^{16}$, AND \\ K. S. WOOD ${ }^{15}$ \\ ApJL accepted
}

\begin{abstract}
We have discovered five millisecond pulsars (MSPs) in a survey of 14 unidentified Fermi-LAT sources in the southern sky using the Parkes radio telescope. PSRs J0101-6422, J1514-4946, and J1902-5105 reside in binaries, while PSRs J1658-5324 and J1747-4036 are isolated. Using an ephemeris derived from timing observations of PSR J0101-6422 $\left(P=2.57 \mathrm{~ms}, \mathrm{DM}=12 \mathrm{pc} \mathrm{cm}^{-3}\right)$, we have detected $\gamma$-ray pulsations and measured its proper motion. Its $\gamma$-ray spectrum (a power law of $\Gamma=0.9$ with a cutoff at $1.6 \mathrm{GeV}$ ) and efficiency are typical of other MSPs, but its radio and $\gamma$-ray light curves challenge simple geometric models of emission. The high success rate of this survey-enabled by selecting $\gamma$ ray sources based on their detailed spectral characteristics - and other similarly successful searches indicate that a substantial fraction of the local population of MSPs may soon be known.
\end{abstract}

Subject headings: gamma rays: general — pulsars: individual (PSR J0101-6422)

\section{INTRODUCTION}

The Large Area Telescope (LAT, Atwood et al. 2009) of the Fermi Gamma-ray Space Telescope is a pulsar detector par excellence. Supported by a radio campaign (Smith et al. 2008) providing ephemerides for coherent folding of LAT photons, the LAT has identified -ray pulsations from many normal (unrecycled) pulsars (e.g. Abdo et al. 2010c) and millisecond pulsars (MSPs, Abdo et al. 2009a). Additionally, tens of new pulsars have been discovered in "blind" searches for periodicity in the LAT data (e.g. Abdo et al. 2009b; Pletsch et al. 2012).

A third, indirect method of detection has been extremely successful in discovering new pulsars, espe-

${ }^{1}$ W. W. Hansen Experimental Physics Laboratory, Kavli Institute for Particle Astrophysics and Cosmology, Department of Physics and SLAC National Accelerator Laboratory, Stanford

University, Stanford, CA 94305, USA

2 email: kerrm@stanford.edu

3 Einstein Fellow

${ }^{4}$ Columbia Astrophysics Laboratory, Columbia University, New York, NY 10027, USA

5 email: fernando@astro.columbia.edu

${ }^{6}$ National Research Council Research Associate, National Acaderny of Sciences, Washington, DC 20001, resident at Naval Research Laboratory, Washington, DC 20375, USA

${ }^{7}$ email: tyrel.j.johnson@gmail.com

${ }^{8}$ NASA Goddard Space Flight Center, Greenbelt, MD 20771, USA

${ }_{9}$ Max-Planck-Institut für Radioastronomie, Auf dem Hügel 69, 53121 Bonn, Germany

10 Netherlands Institute for Radio Astronomy (ASTRON),Postbus 2, 7990 AA Dwingeloo, Netherlands

${ }_{11}$ Astronomical Institute "Anton Pannekoek" University of Amsterdam, Postbus 942491090 GE Amsterdam, Netherlands

${ }_{12}$ CSIRO Astronomy and Space Science, Australia Telescope National Facility, Epping NSW 1710, Australia

${ }^{14}$ National Radio Astronomy Observatory (NRiO), Charlottesville, VA 22903, USA

${ }_{13}$ Jodrell Bank Centre for Astrophysics, School of Physics and Astronomy, The Uni:ersity of Manchester, M13 9PL, UK

${ }^{15}$ Space Science Division, Naval Research Laboratory, Washington, DC 20375-5352, USA

${ }^{16}$ CSIRO Parkes Observatory, Parkes NSW 2870, Australia cially MSPs ${ }^{17}$. Gamma-ray pulsars-both young and recvcled-are stable emitters and nearly all have a characteristic spectrum $d N / d E \propto E^{-\Gamma} \exp \left(-E / E_{c}\right)$ with $\Gamma<2$ and $1<E_{c} / \mathrm{GeV}<10$ (Abdo et al. 2010c), making unidentified, nonvariable LAT sources with such spectra good pulsar candidates. The fine angular resolution and large effective area of the LAT allow a typical source localization of $\sim 10^{\prime}$, about the $1 \mathrm{GHz}$ beam size of $100 \mathrm{~m}$ class radio telescopes. This happy coincidence enables deep pulsation searches with a single pointing. Discovery of a pulsar and a subsequent timing campaign can "close the loop" by providing an ephemeris with which to fold LAT photons and resolve, -ray pulsations. Indeed, since most MSPs reside in binaries, initial detection and characterization of the orbit at longer wavelengths is the only way $\gamma$-ray pulsations can be detected from these pulsars.

Due to sensitivity limitations, LAT-guided surveys naturally target the nearly-isotropic population of nearby MSPs. The deep radio exposures afforded by the efficient target selection offer the tantalizing possibility of completely cataloguing the local field MSPs whose radio and $\gamma$-ray beams cross the Earth. This relatively complete sample will be valuable in constraining the emission mechanisms of MSPs and in characterizing the evolution of their binary progenitors.

Towards this end, we performed a small, targeted survey at the CSIRO Parkes telescope, complementary to a Parkes search described in Keith et al. (2011) that had different source selection criteria.

\section{TARGET SELECTION}

We began with the set of southern (Decl. $<-40^{\circ}$; northern sources are visible to the more sensitive Green Bank Telescope), nonvariable sources in a preliminary version of the 1FGL catalog of $\gamma$-ray sources (Abdo et al.

\footnotetext{
${ }^{17}$ See https://confluence.slac.stanford.edu/display/GLAMCOG/Public+ListDetected+Gamma-Ray+Pulsars for an up-to-date list of LATdetected pulsars
} 
$2010 \mathrm{~b}$ ). We further restricted consideration to sources whose LAT position estimates had a $95 \%$ confidence error radius $\leq 7^{\prime}$, the HWHM of the $1.4 \mathrm{GHz}$ beam of the Parkes telescope, and which had no plausible blazar counterpart. We classified the remaining sources by visual inspection of the $\gamma$-ray spectrum. Sources with spectral shapes resembling known pulsars (see above) were selected for observation, while those sources best described by power laws or with significant spectral breaks but $\Gamma>2$ were deemed likely to be blazars and were not selected. Due to the difficulty of spectral modeling and localization of LAT sources in the Galactic plane, we discarded sources with $|b|<5^{\circ}$. Our final list comprised 14 good candidates. The source positions (see Table 1) were computed from the same analysis used to estimate the spectral shape, but in all cases the difference in position with 1FGL is well within both the 1FGL $95 \%$ error contour and the Parkes beam.

\section{RADIO SEARCHES}

We observed the 14 selected sources $(\S 2)$ at the Parkes $64-\mathrm{m}$ radio telescope between 2009 November 25 and December 8. Each source was observed for betwreen 1 and 2 hours (see Table 1) using the center beam of the multibeam receiver. We recorded 1-bit-digitized total-power samples every $125 \mu$ s from each of 512 frequency channels spanning $256 \mathrm{MHz}$ of band centered on $1390 \mathrm{MHz}$, writing the data to disk for off-line analysis (see Manchester et al. (2001) for more details on the receiver and data-acquisition system.)

The data were analyzed on the koala computer cluster at Columbia University using standard pulsar search techniques implemented in the PRESTO package (Ransom 2001). We dedispersed each data set ideally up to a dispersion measure of $\mathrm{DM}=270 \mathrm{pc} \mathrm{cm}^{-3}$, and when searching for periodic signals we allowed for accelerated signals caused by pulsar motion in a binary system. This was parameterized within PRESTO by the parameter $\mathrm{zmax}=50$, which is related to the maximum number of bins a signai can drift within the Fourier spectrum and stiil be properly detected by the search method. All of the data were analyzed in this manner within about a week of collection, and on 2009 December 4 we found our first MSP. Within a few more days we confirmed it and four other new MSPs. A 6th MSP was detected unbiasedly in our sample, but it had been previously discovered by Keith et al. (2011, see Table 1).

In a few cases, the pulsars were not confirmed on the first attempt and required subsequent observations, owing to their faintness and scintillation in the interstellar medium. We reanalyzed the data by increasing the acceleration search space to $z \max =200$ and by analyzing only the first 30 minutes of each data file. The latter approach improves our sensitivity to highly accelerated pulsars (e.g., for an MSP in a 1-day binary, a 2-hour observation cannot be corrected ideally under the assumption of a constant acceleration, no matter how large the zmax used), but no new pulsars were detected.

The nominal sensitivity of our searches, for a $2 \mathrm{hr}$ observation and assuming a pulsar duty cycle of $20 \%$, is $0.11 \mathrm{mJy}$ for $P \gtrsim 2 \mathrm{~ms}$ and $\mathrm{DM} \lesssim 40 \mathrm{pc} \mathrm{cm}^{-3}$, degrading gradually for shorter periods and larger DMs. While in detail this depends on the sky background temperature, the contribution from the Galaxy at our observing fre- quency only differs by a maximum of $3 \mathrm{~K}$ for different locations, out of a total system equivalent temperature of nearly $30 \mathrm{~K}$, so that we provide an average flux density limit. Two of the non-detections have limits of $0.12 \mathrm{mJy}$ and $0.14 \mathrm{mJy}$ owing to shorter integration times (see Table 1). Because of scintillation, a pulsar with a larger average flux than our nominal limits might not be detectable in a particular observation, and vice versa. In any case, the nominal flux density represents the most sensitive searches for MSPs done at Parkes, especially considering that these LAT-selected targets are largely expected to be relatively nearby.

Characterization of four of the MSPs requires a longer radio timing campaign and/or LAT dataset than available for this work. We defer discussion of these MSPs (and of scintillation effects on sensitivity, which likewise requires repeated observations) to Camilo et al. (in preparation). The remaining pulsar presents a peculiar light curve and we describe it further below.

\section{PSR J0101-6422 \\ 4.1. Timing Solution}

After the discovery observations on 2009 November 25 and confirmation on December 8 , we began regular timing observations of PSR J0101-6422 at Parkes. We observed the pulsar on 35 days through 2011 November 9 , detecting it on 28 occasions; on the remaining 7 days it was too faint to detect, due to interstellar scintillation $\left(\mathrm{DM}=12 \mathrm{pc} \mathrm{cm}^{-3}\right.$.) Each observation was typically one hour, with the same receiver and data acquisition system used in the search observations. Using TEMPO2 ${ }^{18}$ with the 28 times-of-arrival, we obtained a phase-connected timing solution whose parameters are given in Table 2.

The Shklovskii effect (Shklovskii 1970) increases the measured value of $\dot{P}=5.2 \times 10^{-21}$ beyond that intrinsic to the pulsar by $\Delta \dot{P}=P v_{\perp}^{2} / d c$, with $v_{\perp}$ the source velocity transverse to the line-of-sight. For nearby objects, it may dominate the true spindown rate and lead to overestimates of the spindown luminosity (Camilo et al. 1994). The observed proper motion, at the $0.55 \mathrm{kpc} \mathrm{DMI}$ distance (Cordes \& Lazio 2002), implies a true $\dot{P}_{i}=$ $(4.3 \pm 0.1) \times 10^{-21}$. At this distance, the indicated $v_{\perp}=41 \mathrm{~km} \mathrm{~s}^{-1}$ is typical for an MSP (see Nice \& Taylor 1995).

\subsection{Radio Profile and Polarimetry}

We made polarimetric and flux-calibrated observations of PSR J0101-6422, using the PDFB3 digital filterbank. The data vere analyzed with PSRCHIVE (Hotan et al. 2004). The best full-Stokes profile that we obtained is shown in Figure 1 (the flux density of this profile is not necessarily representative of the average pulsar intensity, which varies greatly owing to interstellar scintillation). The main pulse is linearly polarized at the $\sim 15 \%$ level, but the low signal-to-noise ratio prevents a useful determination of rotation measure (the best-fit value is $\sim+10 \mathrm{radm}^{-2}$, but within the uncertainties is consistent with zero). These data show that the subsidiary pulse component is composed of two outer peaks joined by a low bridge of radio emission.

\footnotetext{
18 http://www.atnf.csiro.au/research/pulsar/tempo2
} 
Table 1

Results of Radio Searches of 14 1FGL Sources at Parkes

\begin{tabular}{|c|c|c|c|c|c|c|c|c|c|c|c|}
\hline 1FGL Name & $\begin{array}{c}\text { R.A. }{ }^{\mathrm{a}} \\
(\mathrm{J} 2000.0)\end{array}$ & $\begin{array}{c}\text { Decl. }^{\mathbf{a}} \\
(\mathrm{J} 2000.0)\end{array}$ & $\begin{array}{c}1 \\
(\mathrm{deg})\end{array}$ & $\begin{array}{c}\mathrm{b} \\
(\mathrm{deg})\end{array}$ & $\Gamma$ & $\begin{array}{c}\mathrm{E}_{\mathrm{c}} \\
(\mathrm{GeV})\end{array}$ & $\begin{array}{l}\text { Obs. Time } \\
\text { (hr) }\end{array}$ & $\begin{array}{l}\text { Period } \\
\text { (ms) }\end{array}$ & $\frac{\mathrm{DM}}{\left(\mathrm{pc} \mathrm{cm}^{-3}\right)}$ & $\begin{array}{c}\text { Distance }^{\mathrm{b}} \\
\text { (kpc) }\end{array}$ & Binary \\
\hline J0101.0-6423 & $01^{\mathrm{h}} 00^{\mathrm{m}} 58^{\mathrm{s}} 1$ & $-64^{\circ} 24^{\prime} 03^{\prime \prime}$ & 301.2 & -52.7 & 1.3 & 2.3 & 1.0 & 2.57 & 11.93 & 0.55 & yes \\
\hline J0603.C-4012 & $06^{\mathrm{h}} 03^{\mathrm{m}} 04^{\mathrm{s}} .9$ & $-40^{\circ} 11^{\prime} 02^{\prime \prime}$ & 246.8 & -25.9 & 1.9 & 6.5 & 2.0 & - & - & - & - \\
\hline J0933.9-6228 & $09^{\mathrm{h}} 33^{\mathrm{m}} 58^{\mathrm{s}} .5$ & $-62^{\circ} 27^{\prime} 54^{\prime \prime}$ & 282.2 & -7.8 & 0.1 & 1.2 & 2.0 & - & - & - & - \\
\hline $\mathrm{J} 1036.2-6719$ & $10^{\mathrm{h}} 36^{\mathrm{m}} 16^{\mathrm{s}} 9$ & $-67^{\circ} 20^{\prime} 34^{\prime \prime}$ & 290.4 & -7.8 & 1.5 & 2.2 & 2.0 & - & - & - & - \\
\hline J1227.9-4852 & $12^{\mathrm{h}} 27^{\mathrm{m}} 50 \div 5$ & $-48^{\circ} 51^{\prime} 54^{\prime \prime}$ & 298.9 & 13.8 & 1.8 & 1.6 & 2.0 & - & - & - & - \\
\hline $\mathrm{J} 1232.2-5118$ & $12^{\mathrm{h}} 31^{\mathrm{m}} 49.1$ & $-51^{\circ} 18^{\prime} 50^{\prime \prime}$ & 299.8 & 11.4 & 1.8 & 2.3 & 2.0 & - & - & - & - \\
\hline J1514.1-4945 & $15^{\mathrm{h}} 14^{\mathrm{m}} 05^{\mathrm{s}} 7$ & $-49^{\circ} 45^{\prime} 32^{\prime \prime}$ & 325.2 & 6.8 & 1.7 & 5.9 & 2.0 & 3.59 & 31.5 & 0.9 & yes \\
\hline J1624.0-4041 & $16^{\mathrm{h}} 24^{\mathrm{m}} 06^{\mathrm{s}} \cdot 2$ & $-40^{\circ} 40^{\prime} 48^{\prime \prime}$ & 340.6 & 6.2 & 2.1 & 3.3 & 2.0 & - & - & - & - \\
\hline $\mathrm{J} 1658.8-5317$ & $16^{\mathrm{h}} 58^{\mathrm{m}} 43^{\mathrm{s}} \cdot 2$ & $-53^{\circ} 17^{\prime} 45^{\prime \prime}$ & 335.0 & -6.6 & 2.1 & 1.8 & 1.3 & 2.44 & 30.9 & 0.9 & no \\
\hline J1743.8-7620 & $17^{\mathrm{h}} 43^{\mathrm{m}} 44^{\mathrm{s}} \cdot 6$ & $-76^{\circ} 20^{\prime} 42^{\prime \prime}$ & 317.1 & -22.5 & 1.2 & 2.0 & 1.5 & - & - & - & - \\
\hline $\mathrm{J} 1747.4-4035$ & $17^{\mathrm{h}} 47^{\mathrm{m}} 29.1$ & $-40^{\prime} 36^{\prime} 07^{\prime \prime}$ & 350.2 & -6.4 & 1.5 & 3.4 & 1.4 & 1.65 & 152.9 & 3.3 & no \\
\hline J1902.0-5110 & $19^{\mathrm{h}} 02^{\mathrm{m}} 05^{\mathrm{s}} 5$ & $-51^{\circ} 09^{\prime} 43^{\prime \prime}$ & 345.6 & -22.4 & 1.7 & 4.4 & 1.2 & 1.74 & 36.3 & 1.2 & yes \\
\hline J2039.4-5621 & $20^{\mathrm{h}} 39^{\mathrm{m}} 30^{\mathrm{s}} .5$ & $-56^{\circ} 20^{\prime} 42^{\prime \prime}$ & 341.2 & -37.1 & 1.6 & 2.7 & 1.2 & - & $=$ & - & - \\
\hline $\mathrm{J} 2241.9-5236^{\mathrm{c}}$ & $22^{\mathrm{h}} 41^{\mathrm{m}} 52^{\mathrm{s}} 4$ & $-52^{\circ} 37^{\prime} 37^{\prime \prime}$ & 337.4 & -54.9 & 1.6 & 3.6 & 2.0 & 2.19 & 11 & 0.5 & yes \\
\hline
\end{tabular}

a Parkes telescope pointing position.

b Distances computed from measured DM using the NE2001 model (Cordes \& Lazio 2002).

c See Keith et al. (2011).

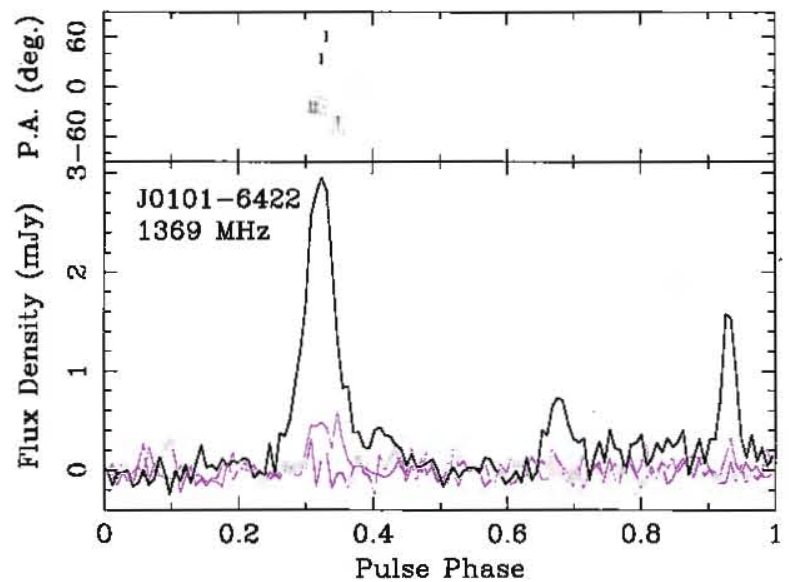

Figure 1. Polarimetric pulse profile of PSR J0101-6422 at $1.4 \mathrm{GHz}$, displayed with 128 phase bins, based on $6 \mathrm{hr}$ of Parkes PDFB3 data using $256 \mathrm{MHz}$ of bandwidth. The black trace corresponds to total intensity, red to linear polarization, blue to circular. In the upper window, the position angle of linear polarization is plotted for bins with linear signal-to-noise ratio $>3$. The pulse peak is displayed with arbitrary phase, and the mean flux density is $\sim 0.2 \mathrm{mJy}$.

\subsection{Gamma-ray Profile}

To characterize the $\gamma$-ray profile, we selected photons collected between 2008 Aug 4 and 2011 Aug 1 with reconstructed energies $0.3-3 \mathrm{GeV}$ lying within $1.1^{\circ}$ of the timing position. This selection balances completeness (including as many pulsed photons as possible) and pulsed signal-to-noise ratio as measured by the H-test (de Jager et al. 1989). We apply the same data processing as described in $\S 4.4$, omitting the horizon cut to increase the livetime by about $20 \%$, at the expense of a slightly increased background.

The light curve corresponding to this extraction appears in Figure 2. If $\gamma$ rays are produced above the null charge surface (the locus of points where the magnetic field is orthogonal to the pulsar spin axis), they appear to an observer in the opposite hemisphere, whereas radio emission from low altitudes is beamed into the same

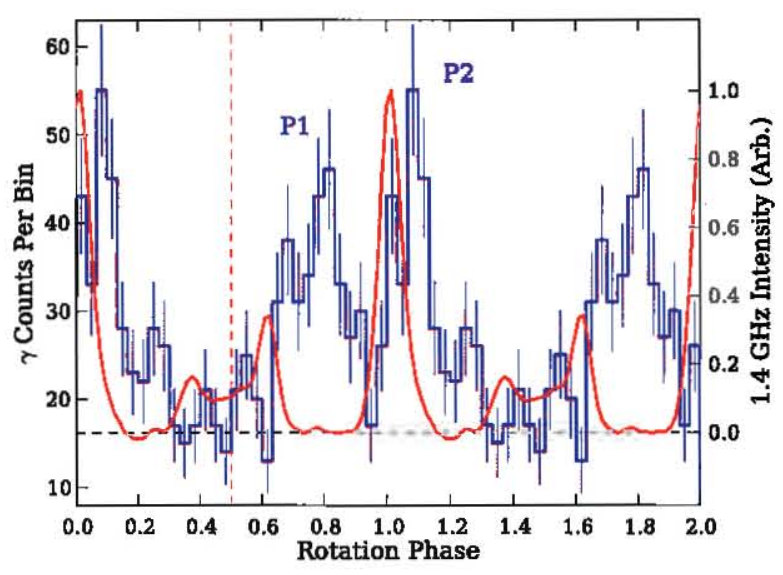

Figure 2. Phase-aligned $\gamma$-ray (blue histogram) and radio (red trace) pulse profiles of PSR J0101-6422. The radio profile is summed from 28 timing detections, while the ' $j$-ray profile corresponds to the optimal aperture described in the main text. The background estimation (dashed horizontal line) is derived by counting photons in the off-pulse phase window (\$4.4) and is consistent with the off-pulse background level obtained via spectral analysis. The vertical dashed line indicates the phase $(\phi \equiv 0.5)$ from which the offset of "P1" is measured.

hemisphere (see Romani \& Yadigaroglu 1995). In this scenario, the line-of-sight to PSR J0101-6422 would intercept a cone of radio emission in one hemisphere to produce the weaker radio peak at $\phi \sim 0.5$ and a cone of radio and $\gamma$ emission in the other to produce the two $\gamma$ peaks and bright radio peak at $\phi \sim 1$. In keeping with this picture and the convention established in the literature, we identify the $\gamma$ peak at $\phi \sim 0.8$ as "P1" and that at $\phi \sim 0.1$ as "P2".

The relative phasing of the peaks is of interest (e.g. Watters et al. 2009). The radio-to- $\gamma$ offset, $\delta$, is measured from the center of the leading radio peak (taken as $\dot{\varphi} \equiv 0.5$, see Figure 2). We estimated the phase of the $\gamma$-ray peaks using unbinned maximum likelihood with a two-sided Lorentzian model for the peaks. These estimates yield $\delta=0.33 \pm 0.01_{\text {stat }} \pm 0.03_{\text {syst }}$ and a $\gamma$-ray peak- 


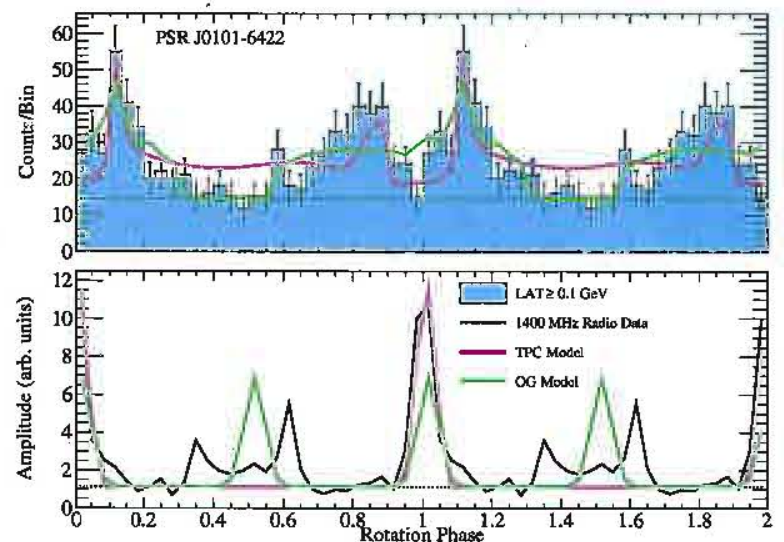

Figure 3: The best-fit model light curves for the two-pole caustic (TPC) and outer gap (OG) models (see §5.1.) The best fit geometries $(\alpha, \zeta)$ for TPC and OG are $\left(26^{\circ}, 79^{\circ}\right)$ and $\left(90^{\circ}, 36^{\circ}\right)$.

to-peal separation of $\Delta=0.26 \pm 0.03_{\text {stat }} \pm 0.02_{\text {syst. }}$. The DM-induced uncertainty in $\delta$ is $<0.001 P$. The systematic uncertainty for both $\delta$ and $\Delta$ is estimated from the scatter obtained by fitting alternative functional forms for the peaks.

\subsection{Gamma-ray Spectrum}

To measure the spectrum of psr, we analyzed low background DIFFUSE class "Pass 6" (Atwood et al. 2009) LAT data collected between 2008 Aug 4 and 2011 Apr 17. We filtered periods where the observatory's rocking angle exceeded $52^{\circ}$ or the Earth's limb impinged upon the field of viev (horizon cut, requires zenith angle $<100^{\circ}$ ). We modelled the sensitivity to these events with the flightcorrected P6_V11_DIFFUSE instrument response function. To compute background contributions, we used a preliminary version of the 2FGL catalog and its accompanying diffuse emission models ${ }^{19}$. We selected events within $10^{\circ}$ and re-fit the spectra of point sources within $8^{\circ}$ and the no:malizations of the diffuse models. Fits employed pointliive (Kerr 2011), a binned maximum likelihood algorithm.

The phase-averaged spectrum of PSR J0101-6422 is well described by an exponentially cutoff power law, $d N / d E=N_{0}\left(E / E_{0}\right)^{-\Gamma} \exp \left(-E / E_{c}\right)$, whose parameters appear in Table 2. We also considered separately the spectra of the three major components, viz. P2 $(0<\phi<0.33)$, the off-pulse $(0.33<\phi<0.63)$, and P1 $(0.63<\phi<1)$. The parameter uncertainties are relatively large, and accordingly we found no significant difference in the spectral shapes of the two peaks. Further, we detected no significant emission in the off-pulse phase window and derived a 95\% confidence upper limit on the energy flux of a point source with a power law spectrum with $\Gamma=2.2$, obtaining $6 \times 10^{-12} \mathrm{erg} \mathrm{cm}^{-2}$ $\mathrm{s}^{-1}$ (> $100 \mathrm{MeV}$; scaled up by 3.3 to the full phase window.)

The observed flux is related to the $\gamma$-ray luminosity by $F_{\gamma}=L_{\gamma} / 4 \pi f_{\Omega} d^{2}$, where $f_{\Omega}$ is an unknown beaming factor typically of order unity, e.g. Watters et al. 2009) implying a phase-averaged, isotropic $\left(f_{\Omega}=1\right) \gamma$-ray luminosity $L_{\gamma}=4.0 \times 10^{32} \mathrm{erg} \mathrm{s}^{-1}=0.04 \dot{E}$ at $d=0.55$

\footnotetext{
produce the radio interpulse and produces too much off-
${ }^{19} \mathrm{http}: / /$ fermi.gsfc.nasa.gov/ssc/data/access/lat/BackgroundModels.
}

kpc. Best-fit values of $f_{\Omega}$ from the TPC and OG models $(\S 5.1)$ are 0.7 and 0.9 . This $\gamma$-ray efficiency is in accord with other LAT-detected MSPs (Abdo et al. 2009a).

\section{5. $X$-ray and Optical Observations}

A $3.3 \mathrm{ks}$ Swift observation with the X-ray Telescope in PC mode was obtained on 2009 Nov 21 to search for an $\mathrm{X}$-ray counterpart to the then-unidentified 1FGL source. No significant counterpart is detected, with a $3 \sigma$ upper limit at the pulsar position of $2.0 \times 10^{-3} \mathrm{cts} \mathrm{s}^{-1}(0.5-8$ $\mathrm{keV}$ ). Assuming a power-law spectrum with photon index $\Gamma=1.5$ for a column with $N_{\mathrm{H}}=3.7 \times 10^{20} \mathrm{~cm}^{-2}(10$ $\mathrm{HI}$ atoms per free electron along the line-of-sight) yields an unabsorbed flux limit of $1.1 \times 10^{-13} \mathrm{erg} \mathrm{cm}^{-2} \mathrm{~s}^{-1}$, or efficiency $L_{X} / \dot{E}<5.0 \times 10^{-4}$ at $0.55 \mathrm{kpc}$. This limit is comparable to the detected X-ray flux from other MSPs (e.g. Marelli et al. 2011).

We searched four DSS plates and found no optical counterpart consistent with the pulsar's position, suggesting $m>21$.

\section{DISCUSSION}

\subsection{PSR J0101-6422}

At first blush, PSR J0101-6422 is an unremarkable member of the growing population of $\gamma$-ray MSPs. The orbital characteristics suggest an epoch of Roche lobe overflow from an evolved $1-2 M_{\odot}$ companion which later became the $\sim 0.2 M_{\odot}$ He white dwarf currently inferred in the system (e.g. Tauris \& Savonije 1999). However, as we discuss below, PSR J0101-6422's light curve is challenging to explain with simple geometric models that tie the radio and $\gamma$ emission to particular regions of the magnetosphere.

Remarkably, a simple picture of $\gamma$-ray emission arising in the outer magnetosphere appears to describe the majority of both young (though see Romani et al. 2011) and recycled pulsars (e.g. Venter et al. 2009). On the other hand, while radio emission in young pulsars and some MSPs appears to come from lower altitudes, several MSPs have phase-aligned $\gamma$ and radio peaks (e.g. Abdo et al. 2010a), implying a joint emission site at high altitude. These observations raise the prospect of MSPs whose radio emission combines "traditional" polar cap emission with a higher-altitude component.

To determine which scenario best describes the emission pattern of PSR J0101-6422, we performed joint fits to the radio and $\gamma$-ray data following the method of Johnson (2011). We modelled the radio as a simple "cone" component (Story et al. 2007) and the r rays using both the "two-pole caustic" (TPC) model of Dvks \& Rudak (2003, N.B. we adopt a larger maximum cylindrical radius of 0.95 of the light cylinder) and the "outer gap" (OG) model (Cheng et al. 1986; Romani \& Yadigaroglu 1995). The angle between the pulsar spin axis and the line-of-sight, $\zeta$, is unknown $a$ priori, but if the radio emission arises from low altitudes, then the presence of two peaks separated by roughly half of a rotation indicates the inclination of the magnetic axis from the spin axis $(\alpha)$ cannot be small. The best-fit light curves for the two $\gamma$-ray models appear in Figure 3. The low $\alpha=26^{\circ}$ preferred for the TPC model cannot 
Table 2

Measured and Derived Parameters for PSR J0101-6422

\begin{tabular}{|c|c|}
\hline Parameter & Value \\
\hline 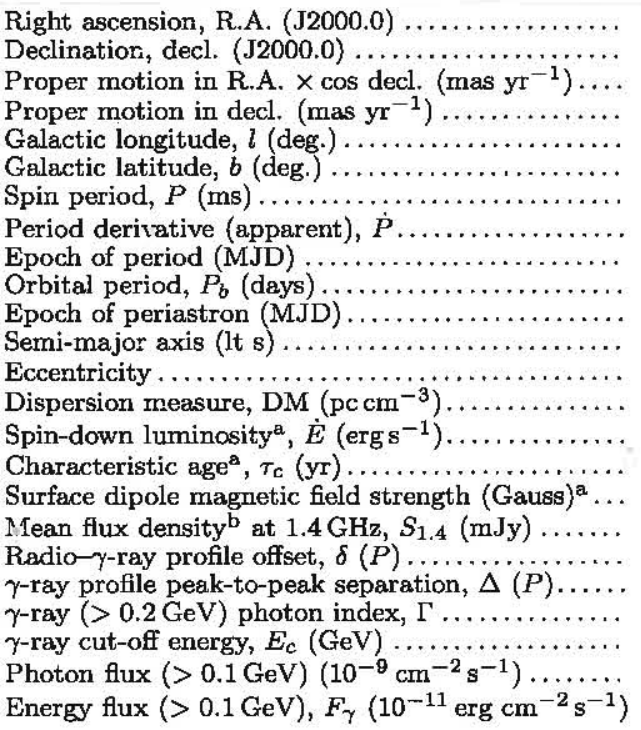 & $\begin{array}{l}01^{\mathrm{h}} 01^{\mathrm{m}} 11^{\mathrm{s}} 1163(2) \\
-64^{\circ} 22^{\prime} 30^{\prime \prime} 171(2) \\
10(1) \\
-12(2) \\
301.19 \\
-52.72 \\
2.5731519721683(2) \\
5.16(3) \times 10^{-21} \\
55520 \\
1.787596706(2) \\
55162.4011764(3) \\
1.701046(2) \\
<1.5 \times 10^{-5} \\
11.926(1) \\
1.0 \times 10^{34}-16 \% \\
9 \times 10^{9}+20 \% \\
1.1 \times 10^{7}-9 \% \\
0.28 \pm 0.06 \\
0.35 \pm 0.01 \pm 0.05 \\
0.26 \pm 0.03 \pm 0.02 \\
0.9 \pm 0.3 \pm 0.2 \\
1.6 \pm 0.4 \pm 0.3 \\
9.5 \pm 1.8-2.5 \\
1.1 \pm 0.1 \pm 0.1\end{array}$ \\
\hline
\end{tabular}

Note. - Timing solution parameters given in TDB relative to the DE405 planetary ephemeris. Numbers in parentheses represent the measured $1 \sigma$ TENIPO2 timing uncertainties on the last digits quoted. For $\gamma$-ra parameters, the first uncertainty is statistical and the second systematic.

a Corrected for Shklovskii effect at $0.55 \mathrm{kpc}$; the percentage change from the nominal value is given.

b Arerage flux for 28 timing detections.

$\alpha=90^{\circ}$ (orthogonal rotator), produces two radio peaks but fails to produce two clear $\gamma$ peaks and the radio interpulse morphology.

Since neither model faithfully reproduces the observed light curves, we propose that one or both of the radio peaks may originate at higher altitude. Emission over a range of altitudes would also explain the low observed linear polarization of PSR J0101-6422 (e.g. Dyks et al. 2004) \&nd admit smaller $\alpha$ values. Alternativelv, the failure of geometric models may indicate that the physical details of the MSP magnetosphere- e.g., currents, multipolar fields, and plasma loading of the field lines-and the radio and $\gamma$ emission mechanisms play an important role.

\subsection{Survey Implications}

With 6/14 pointings resulting in unbiased MSP detections, this surrey was fruitful, though this high efficiency may be luck. Indeed, in our timing campaign, we only detected PSR J0101-6422 on 28 of 35 attempts, and several observations required prior knowledge of the ephemeris for detection. Thus, because of scintillation, we estimate that PSR J0101-6422 is only detectable in $2 / 3$ observations, depending somewhat on integration time. Many field MiSPs are in eclipsing binaries, further diminishing the probability of detection in a single observation.

We believe the strongest factor in our search's success was the source selection criteria. E.g., Ransom et al. (2011) selected nonvariable LAT sources (but including no spectral shape information) and found MSPs in $3 / 25$ pointings. But when considering only targets with $|b|>5^{\circ}$, their efficiency jumps to $3 / 8$. Keith et al. (2011) employed some spectral information and detected MSPs unbiasedly in $1 / 11$ pointings $\left(1 / 4\right.$ for $\left.|b|>5^{\circ}\right)$. A short list of 7 spectrally-exceptional candidates drawn up for a Nançay radio telescope survey included 5 MSPs, three of which were discovered from Nançay observations (Cognard et al. 2011; Guillemot et al. 2011). And, Hessels et al. (2011) used nearly identical selection criteria as this work in a Green Bank Telescope survey at 350 $\mathrm{MHz}$ and found MSPs in 13/49 pointings, all but one at $|b|>5^{\circ}$.the

Targeting pulsar-like sources is clearly efficient. However, new LAT sources vill lie at the sensitivity threshold and the limited statistics will not admit classification of spectral shape and variability. Nonetheless, we argue that future radio searches should omit detailed ranking schemes and target any LAT source off the Galactic plane that has not been associated with a known blazar.

We believe this substantially increased target list (and telescope time) is justified. In the 2FGL source catalog (Abdo et al. 2011), there are $\sim 350$ unassociated sources more than $5^{\circ}$ off the Galactic plane. MSPs account for roughly $5 \%$ of associated LAT sources, so $15-20$ MSPs may remain to be found in these unassociated 2FGL sources. With only 350 positions to monitor, multiple deep observations can ameliorate the confounding effects of scintillation and eclipses while requiring a fraction of the time of a comparabiy complete all-sky survey. By comparison, the otherwise prodigious Parkes multibeam survey found "only" 17 MSPs in over 40,000 pointings (e.g. Faulkner et al. 2004). In addition to the intrinsic 
interest in discovering new MSPs, by thoroughly searching all such LAT sources, we will gain confidence that the MSPs we have found can truly provide a volume-limited sample of the energetic, $\gamma$-loud MSPs, an invaluable step in the study of the MSP population of the Milky Way.

We thank the marvelous staff at Parkes that make it such a wonderful research facility, Willem van Straten for help with PSRCHIVE, and Jules Halpern for help with optical analysis. The Parkes Observatory is part of the Australia Telescope, which is funded bv the Commonwealth of Australia for operation as a National Facility managed by CSIRO.

The Fermi LAT Collaboration acknowledges support from a number of agencies and institutes for both development and the operation of the LAT as well as scientific data analysis. These include NASA and DOE in the United States, CEA/Irfu and IN2P3/CNRS in France, ASI and INFN in Italy, MEXT, KEK, and JAXA in Japan, and the K. A. Wallenberg Foundation, the Swedish Research Council and the National Space Board in Sweden. Additional support from INAF in Italy and CNES in France for science analysis during the operations phase is also gratefully acknowledged.

Support for this work was provided by NASA through Einstein Postdoctoral Fellowship Award Number PF0110073 issued by the Chandra X-ray Observatory Center, which is operated by the SAO for and on behalf of NASA under contract NAS8-03060.

Facilities: Fermi (LAT), Parkes (PMDAQ, PDFB3)

\section{REFERENCES}

Abdo, A. A., et al. 2009a, Science, 325, 848
- $2009 \mathrm{~b}$, Science, 325,840

-. 2010a, ApJ, 712, 957

-. 2010b, ApJS, 188, 405

-. 2010c, ApJS, 187,460

—. 2011, arXiv: 1108.1435

Atwood, W. B., et al. 2009, ApJ, 697, 1071

Camilo, F., Thorsett, S. E., \& Kulkarni, S. R. 1994, ApJ, 421, L15

Cheng, K. S., Ho, C., \& Ruderman, M. 1986, ApJ, 300, 500

Cognard, I., et al. 2011, ApJ, 732, 47

Cordes, J. M., \& Lazio, T. J. WV. 2002, ArXiv Astrophysics e-prints arXiv:astro-ph/0207156

de Jager, O. C., Raubenheimer, B. C., \& Swanepoel, J. W. H. 1989, A\&A, 221, 180

Dyks, J., Harding, A. K., \& Rudak, B. 2004, ApJ, 606, 1125

Dyks, J., \& Rudak, B. 2003, ripJ, 598, 1201

Faulkner, $\dot{x}$. J., et al. 2004, MNRAS, 355, 147

Guillemot, L., et al. 2011, MNRAS, submitted

Hessels, J. W. T., et al. 2011, arXiv:1101.1742

Hotan, A. W., van Straten, W., \& Manchester, R. N. 2004, Publications of the Astron. Soc. of Australia, 21, 302

Johnson, T. 2011, $\mathrm{PhD}$ Thesis, University of Maryland, arXiv:TBD

Keith, M. J., et al. 2011, MNRAS, 414, 1292

Kerr, M. 2011, $\mathrm{PhD}$ Thesis, University of Washington, arXiv:1101.6072

Manchester, R. N., et al. 2001, MNRAS, 328, 17

Marelli, M., De Luca, A., \& Caraveo, P. A. 2011, ApJ, 733, 82

Nice, D. J., \& Taylor, J. H. 1995, ApJ, 441, 429

Pletsch, H. J., et al. 2012, ApJ, 744, 105

Ransom, S. M. 2001, PhD thesis, Harvard University

Ransom, S. M., et al. 2011, ApJ, 727, L16

Romani, R. W., Kerr, M., Craig, H. A., Johnston, S., Cognard, I., \& Smith, D. A. 2011, ApJ, 738, 114

Romani, R. W., \& Yadigaroglu, I.-A. 1995, ApJ, 438, 314

Shklovskii, I. S. 1970, Soviet Ast., 13, 562

Smith, D. A., et al. 2008, A\&A, 492, 923

Story, S. A., Gonthier, P. L., \& Harding, A. K. 2007, ApJ, 671, 713

Tauris, T. M., \& Savonije, G. J. 1999, A\&A, 350, 928

Venter, C., Harding, A. K., \& Guillemot, L. 2009, ApJ, 707, 800

Watters, K. P., Romani, R. W., Weltevrede, P., \& Johnston, S. 2009, ApJ, 695, 1289 